TRANSACTIONS OF THE

AMERICAN MATHEMATICAL SOCIETY

Volume 358, Number 12, December 2006, Pages 5523-5538

S 0002-9947(06)03893-1

Article electronically published on July 21, 2006

\title{
SINGULARITIES OF LINEAR SYSTEMS AND THE WARING PROBLEM
}

\author{
MASSIMILIANO MELLA
}

\begin{abstract}
The Waring problem for homogeneous forms asks for additive decomposition of a form $f$ into powers of linear forms. A classical problem is to determine when such a decomposition is unique. In this paper we answer this question when the degree of $f$ is greater than the number of variables. To do this we translate the algebraic statement into a geometric one concerning the singularities of linear systems of $\mathbb{P}^{n}$ with assigned singularities.
\end{abstract}

\section{INTRODUCTION}

Edward Waring stated in 1770 that every integer is a sum of at most 9 positive integral cubes, also a sum of at most 19 biquadrates, and so on. Later on Jacobi and others considered the problem of finding all the decompositions of a given number into the least number of powers, Di]. In this paper we are concerned with a similar question for general homogeneous forms.

Let $f$ be any form of degree $d$ in $n+1$ variables over the complex numbers. It is easy to prove that $f$ can be decomposed additively in powers of linear forms. That is, $f=l_{1}^{d}+\ldots+l_{s}^{d}$ for $l_{i}$ linear forms. Indeed this is equivalent to the nondegeneracy of the Veronese embedding $\nu_{d}\left(\mathbb{P}^{n}\right) \subset \mathbb{P}\left(S_{d}\right)$, where $S_{d}$ is the vector space of degree $d$ polynomials in $\mathbb{C}\left[x_{0}, \ldots, x_{n}\right]$. Moreover the minimum $s$ for which $\operatorname{Sec}_{s-1}\left(\nu_{d}\left(\mathbb{P}^{n}\right)\right)=\mathbb{P}\left(S_{d}\right)$ gives the least number of factors in the decomposition of a general form $f$. It is now well known that Alexander-Hirschowitz' result, [AH2], allows us to give a statement, similar to Waring's, for the additive decomposition of general homogeneous forms. This was first noticed by Iarrobino, Ia], using apolarity and inverse systems. The way we prefer to look at it is through the Terracini lemma.

As Jacobi did for the original Waring problem, the Waring problem for forms has also been investigated in an attempt to understand all possible decompositions of a given form. By means of Hilbert schemes or Grassmannians one can give the structure of an algebraic variety to the set of decompositions of a given form. In this way Mukai gave a description of the Fano 3-fold $V_{22}$ as the variety representing all decompositions of a plane quartic, $\mathrm{Mu}$. Iliev, Ranestad and Schreyer applied similar arguments to other special cases, RS, IR1 and IR2. Classic works of Sylvester, Reye, Richmond, Hilbert and Palatini investigated the case in which the

Received by the editors June 17, 2004 and, in revised form, November 17, 2004.

2000 Mathematics Subject Classification. Primary 14J70; Secondary 14N05, 14E05.

Key words and phrases. Waring, linear system, singularities, birational maps.

This work was partially supported by Progetto Cofin 2002 "Geometria sulle varietà algebriche" Miur, Eager.

(C)2006 American Mathematical Society Reverts to public domain 28 years from publication 
decomposition is unique. More recently Iarrobino and Kanev treated the same problem for special forms, [IK]; see Remark 4.6. The following list is taken almost verbatim from [RS]. A general form $f$ of degree $d$ in $n+1$ variables has a unique presentation as a sum of $s$ powers of linear forms in the following cases:

- $n=1, d=2 k-1$ and $s=k[\mathrm{Sy}$,

- $n=3, d=3$ and $s=5$ Sylvester's Pentahedral Theorem [Sy],

- $n=2, d=5$ and $s=7$ [Hi], [Ri], [Pa].

In this paper we prove that if $d>n$ there are no further examples. More precisely we have the following.

Theorem 1. Let $f$ be a general homogeneous form of degree $d$ in $n+1$ variables. Assume that $d>n>1$. Then $f$ is expressible as a sum of $d$ th powers of linear forms in a unique way if and only if $n=2$ and $d=5$. This unique exception is well known classically [Hi].

To prove Theorem 1 we use the geometric interpretation sketched before. In particular we translate the uniqueness assumption into a statement about singularities of linear systems with imposed singularities. Next we study the singularities of this linear systems. This is an interesting problem in itself. Let us introduce it. Consider integers $(d, n, l)$. One wants to understand the singularities of a general hypersurface $F$ of degree $d$ that is singular at $l$ general points of the projective space $\mathbb{P}^{n}$. We expect that when $l$ is as high as possible there is no room, in general, for other singularities. That is, $F$ has only ordinary double points at the imposed singularities, and it is smooth elsewhere. On the other hand if this is true for the highest $l$, then it is a fortiori true for lower impositions. Therefore one can expect that, with few exceptions, $F$ is irreducible, and it has only $l$ ordinary double points. For $\mathbb{P}^{2}$ this has been classically studied, and it is the content of a theorem of Arbarello-Cornalba, $\mathrm{AC}$.

The starting point, to extend Arbarello-Cornalba's result to a higher dimension, are the beautiful papers of Alexander-Hirschowitz, AH1 AH2. To reach our goal we will base our work not only on their result but also on their degeneration idea. Indeed to determine the singularities of $F$ one can consider a suitable degeneration of it. This is done, in the full spirit of $\mathrm{A}-\mathrm{H}$, by specializing a group of points to a hyperplane. Then an infinitesimal Bertini Theorem, $\mathrm{CC}$, similar to A-C's argument for plane curves, allows us to determine the singularities on $F$.

The $\mathrm{A}-\mathrm{H}$ technique consists in choosing numbers in a clever way. In such a way one can prove the statement by a double induction on degree and dimension. Furthermore when the numbers are not amicable they use the differentiable Horace Lemma to pop out some points from the hyperplane back to $\mathbb{P}^{n}$. Unfortunately we are not able to apply the Horace Lemma, because we lose control on the singularities of the specialized linear system. Furthermore singularities behave better in a degree induction than in a dimensional one. For these reasons we have to develop a slightly different approach. We play the full induction on the degree when $l$ is "small"; see Theorem 4.1. Then the result needed for Theorem[1, and also a bit more, is obtained in one step using the small $l$ case for degree $d-1$; see Theorem 4.3. The latter has an application to weakly defectiveness of the Veronese embedding, Corollary 4.5.

Here is an outline of the paper. In section 1 we introduce the main notations, definitions and results that will be used throughout the paper. In particular a lemma on the upper semi-continuity of the dimension of singularities is established. 
In section 2 we explain how to pass from uniqueness in the Waring problem to a singularity statement. In doing this we show the rationality of varieties with one apparent $(k+1)$-tuple secant $\mathbb{P}^{k}$, improving on results of [CMR]; see also [CR].

In section 3 we prove the main numerology to speed up the induction of the main theorem about singularities of linear systems. Section 4 is the core of the induction and contains the main result on singularities. Finally we prove Theorem 1 .

Work began on this problem during a short visit at Università Tor Vergata in Roma. It is a pleasure to thank Ciro Ciliberto and Francesco Russo for discussions, motivations and interest in this project.

\section{Notation AND PRELIMINARIES}

Unless otherwise stated we work over the field of complex numbers. First we introduce what is needed to study linear systems with prescribed singularities.

Definition 1.1. Let $p \in \mathbb{P}^{n}$ be a point. The double point at $p$ in $\mathbb{P}^{n}$ is the scheme given by the square of the ideal sheaf of $p$. If $P \subset \mathbb{P}^{n}$ is a collection of points, we denote by $P^{2}$ the double points supported on $P$. In particular the linear system $\left|\mathcal{I}_{P^{2}}(d)\right|$ is given by hypersurfaces of degree $d$ singular at $P$.

Given a collection of points $X=P \cup Q$, with $Q$ supported on a hyperplane $H$, let $\tilde{X}$ be the residual of $X^{2}$ with respect to $H$. That is, $\tilde{X}=P^{2} \cup Q$. Then there is the Castelnuovo exact sequence given by

$$
0 \rightarrow \mathcal{I}_{\tilde{X}}(d-1) \rightarrow \mathcal{I}_{X^{2}}(d) \rightarrow \mathcal{I}_{Q^{2}, H}(d) \rightarrow 0 .
$$

This gives the following sequence on cohomology:

$$
0 \rightarrow H^{0}\left(\mathbb{P}^{n}, \mathcal{I}_{\tilde{X}}(d-1)\right) \rightarrow H^{0}\left(\mathbb{P}^{n}, \mathcal{I}_{X^{2}}(d)\right) \rightarrow H^{0}\left(H, \mathcal{I}_{Q^{2}}(d)\right) .
$$

Definition 1.2. Consider a collection $P$ of $l$ general points in $\mathbb{P}^{n}$. Define

$$
\mathcal{G}_{d, n, l}:=\left|\mathcal{I}_{P^{2}}(d)\right| \text {. }
$$

Fix a hyperplane $H$ and a collection $X=P \cup Q$, where $P$ is given by $(l-h)$ general points in $\mathbb{P}^{n}$, and $Q=\bigcup_{1}^{h} q_{j}$ is given by $h$ general points in $H$. Define

$$
\mathcal{H}_{H, d, n, l, h}:=\left|\mathcal{I}_{X^{2}}(d)\right| \text {. }
$$

In this paper we are interested in nonempty linear systems of type $\mathcal{G}_{d, n, l}$. For this we introduce the following definition.

Definition 1.3. We say that the linear system $\mathcal{G}_{d, n, l}$ is expected if

$$
\operatorname{dim} \mathcal{G}_{d, n, l}=\left(\begin{array}{c}
n+d \\
n
\end{array}\right)-(n+1) l-1
$$

Moreover if $\mathcal{G}_{d, n, l}$ is expected and $\operatorname{dim} \mathcal{G}_{d, n, l} \geq 0$, we say that it is expected and effective.

Note that if $\mathcal{G}_{d, n, l}$ is expected and effective, then $\mathcal{G}_{d, n, l^{\prime}}$ is expected and effective for any $l^{\prime}<l$. Similarly for linear systems of type $\mathcal{H}$. We say that $\mathcal{H}_{H, d, n, l, h}$ is expected and effective if

$$
\operatorname{dim} \mathcal{H}_{H, d, n, l, h}=\left(\begin{array}{c}
n+d \\
n
\end{array}\right)-(n+1) l-1 \geq 0
$$

Note that if $\mathcal{H}_{H, d, n, l, h}$ is expected and effective, then by semi-continuity $\mathcal{G}_{d, n, l}$ is expected and effective. In the following we frequently ask expected and effective 
linear systems of type $\mathcal{H}$ to satisfy the following further properties. The linear system $\mathcal{H}_{H, d, n, l, h}$ is what I need (wIn) if

- $\mathcal{H}_{H, d, n, l, h}$ is expected and effective,

- $\left|\mathcal{H}_{H, d, n, l, h} \otimes \mathcal{I}_{H}\right| \neq \emptyset$,

- (1) is a short exact sequence.

The following is a weak form of the main result of $\mathrm{AH} 2$.

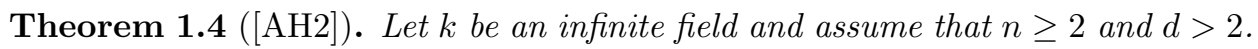
Then $\mathcal{G}_{d, n, l}$ is expected and effective if $l<\left\lceil\frac{\left(\begin{array}{c}d+n \\ n\end{array}\right)}{n+1}\right\rceil$.

Furthermore if $\operatorname{dim} \mathcal{G}_{d, n, l} \geq 0$ and $\mathcal{G}_{d, n, l}$ is not expected and effective, then $l=$ $\left\lceil\frac{\left(\begin{array}{c}n+d \\ n\end{array}\right)}{n+1}\right\rceil, \operatorname{dim} \mathcal{G}_{d, n, l}=0$, and $(d, n, l)=(3,4,7),(4,2,5),(4,3,9),(4,4,14)$.

The aim is to prove the statement on singularities by degeneration. To do this we need to control how the singularities behave under specializations. This is the content of the next lemma and corollary.

Lemma 1.5. Let $\Delta$ be a complex disk around the origin. Consider the product $V=\mathbb{P}^{n} \times \Delta$, with the natural projections $\pi_{1}$ and $\pi_{2}$. Let $V_{t}=\mathbb{P}^{n} \times\{t\}$ and $\mathcal{O}_{V}(d)=\pi_{1}^{*}\left(\mathcal{O}_{\mathbb{P}^{n}}(d)\right)$. Fix a configuration $p_{1}, \ldots, p_{l}$ of $l$ points on $V_{0}$ and let $\sigma_{i}$ : $\Delta \rightarrow V$ be sections such that $\sigma_{i}(0)=p_{i}$ and $\left\{\sigma_{i}(t)\right\}_{i=1, \ldots, l}$ are general points of $V_{t}$ for $t \neq 0$. Let $P_{t}=\bigcup_{i=1}^{l} \sigma_{i}(t)$.

Consider the linear system $\mathcal{H}_{t}=\left|\mathcal{O}_{V_{t}}(d) \otimes \mathcal{I}_{P_{t}^{2}}\right|$. Assume that $l<\left\lceil\frac{\left(\frac{d+n}{n+1}\right)}{n+1}\right\rceil$ and $\operatorname{dim} \mathcal{H}_{0}=\operatorname{dim} \mathcal{H}_{t}$, for $t \in \Delta$. Let $\varphi_{i}(t):=\operatorname{dim}_{\sigma_{i}(t)} \operatorname{Sing} \mathcal{H}_{t}$ and $\psi_{i}(t):=$ $\operatorname{dim}_{\sigma_{i}(t)} \operatorname{Bsl} \mathcal{H}_{t}$. Then for $t \neq 0$, we have

$$
\varphi_{i}(t) \leq \min \left\{j \mid \varphi_{j}(0)\right\}
$$

and

$$
\psi_{i}(t) \leq \min \left\{j \mid \psi_{j}(0)\right\} .
$$

Proof. Let $\xi_{i}$ be the generic point of $\sigma_{i}(\Delta)$ and $P=\bigcup_{i=1}^{l} \xi_{i}$. Let $\mathcal{H}=\left|\mathcal{O}_{V}(d) \otimes \mathcal{I}_{P^{2}}\right|$. Then by Theorem 1.4 applied to the field $\mathbb{C}(t)$, we have

$$
\operatorname{dim} \mathcal{H}=\operatorname{dim} \mathcal{H}_{t}=\left(\begin{array}{c}
n+d \\
n
\end{array}\right)-(n+1) l-1 .
$$

Let $D \in \mathcal{H}$ be a general element; then $D_{\mid V_{t}}$ is a general element in $\mathcal{H}_{t}$. In particular Bsl $\mathcal{H}_{\mid V_{t}}=\operatorname{Bsl} \mathcal{H}_{t}$ and we can assume, by the Bertini Theorem, that Sing $\mathcal{H}_{t}=\operatorname{Sing} \mathcal{H} \cap V_{t}$, for $t \neq 0$. The former, together with the semi-continuity of the dimension of fibers, show that $\psi_{i}(t) \leq \psi_{i}(0)$. The variety $V_{t}$ is a Cartier divisor, therefore

$$
\operatorname{dim}_{\sigma(0)}\left(\operatorname{Sing} \mathcal{H} \cap V_{0}\right)=\operatorname{dim}_{\sigma(t)}\left(\operatorname{Sing} \mathcal{H} \cap V_{t}\right) .
$$

This gives

$$
\varphi_{i}(t)=\operatorname{dim}_{\sigma_{i}(t)}\left(\operatorname{Sing} \mathcal{H} \cap V_{t}\right)=\operatorname{dim}_{\sigma_{i}(0)}\left(\operatorname{Sing} \mathcal{H} \cap V_{0}\right) \leq \operatorname{dim}_{\sigma_{i}(0)} \operatorname{Sing} \mathcal{H}_{0}=\varphi_{i}(0) .
$$

To conclude, observe that since the points are general, a monodromy argument shows that $\varphi_{i}(t)=\varphi_{j}(t)$, for $t \neq 0$, and similarly for functions $\psi_{i}$.

Corollary 1.6. Assume that $\mathcal{H}_{H, d, n, l, h}$ is wIn and there exists $D \in \mathcal{H}_{H, d, n, l, h}$ with isolated singularities at some point $p \in P$. Then the general element $G \in \mathcal{G}_{d, n, l}$ has only ordinary double points. 
Proof. The linear system $\mathcal{H}_{H, l, d, n, h}$ is wIn. In particular there exists a degeneration like the one described in Lemma 1.5 with $\mathcal{H}_{0}=\mathcal{H}_{H, l, d, n, h}$. The lemma ensures that $\operatorname{dim} \operatorname{Sing} G=0$. Then by [CC, Theorem 1.4] $G$ has only ordinary double points.

Let us recall, next, the main definitions and results concerning secant varieties. Let $G_{k}=G(k, N)$ be the Grassmannian of $k$-linear spaces in $\mathbb{P}^{N}$. Let $X \subset \mathbb{P}^{N}$ be an irreducible variety

$$
\Gamma_{k}(X) \subset X \times \cdots \times X \times G_{k}
$$

the closure of the graph of

$$
\alpha:(X \times \cdots \times X) \backslash \Delta \rightarrow G_{k},
$$

taking $\left(x_{0}, \ldots, x_{k}\right)$ to the $\left[\left\langle x_{0}, \ldots, x_{k}\right\rangle\right]$, for $(k+1)$-tuple of distinct points. Observe that $\Gamma_{k}(X)$ is irreducible of dimension $(k+1) n$. Let $\pi_{2}: \Gamma_{k}(X) \rightarrow G_{k}$ be the natural projection. Denote

$$
S_{k}(X):=\pi_{2}\left(\Gamma_{k}(X)\right) \subset G_{k} .
$$

Again $S_{k}(X)$ is irreducible of dimension $(k+1) n$. Finally let

$$
I_{k}=\{(x, \Lambda) \mid x \in \Lambda\} \subset \mathbb{P}^{N} \times G_{k},
$$

with natural projections $\pi_{i}$ onto the factors. Observe that $\pi_{2}: I_{k} \rightarrow G_{k}$ is a $\mathbb{P}^{k}$-bundle on $G_{k}$.

Definition 1.7. Let $X \subset \mathbb{P}^{N}$ be an irreducible variety. The abstract $k$-Secant variety is

$$
\operatorname{Sec}_{k}(X):=\pi_{2}^{-1}\left(S_{k}(X)\right) \subset I_{k}
$$

while the $k$-Secant variety is

$$
\operatorname{Sec}_{k}(X):=\pi_{1}\left(\operatorname{Sec}_{k}(X)\right) \subset \mathbb{P}^{N} .
$$

It is immediate that $\operatorname{Sec}(X)$ is a $((k+1) n+k)$-dimensional variety with a $\mathbb{P}^{k}$-bundle structure on $S_{k}(X)$. One says that $X$ is $k$-defective if

$$
\operatorname{dim} \operatorname{Sec}_{k}(x)<\min \left\{\operatorname{dim} \operatorname{Sec}_{k}(X), N\right\} .
$$

Remark 1.8. A feature of the above definition is the following simple observation. Let $\Lambda_{1}$ and $\Lambda_{2}$ be two $(k+1)$-secant $k$-linear spaces to $X \subset \mathbb{P}^{N}$. Let $\lambda_{1}$ and $\lambda_{2}$ be the corresponding projective $k$-spaces in $\operatorname{Sec}_{k}(X)$. Then we have $\lambda_{1} \cap \lambda_{2}=\emptyset$.

Here is the main result we use regarding secant varieties.

Theorem 1.9 (Terracini Lemma [Te,, $\mathrm{CC}]$ ). Let $X \subset \mathbb{P}^{N}$ be an irreducible, projective variety. If $p_{0}, \ldots, p_{k} \in X$ are general points and $z \in\left\langle p_{0}, \ldots, p_{k}\right\rangle$ is a general point, then the embedded tangent space at $z$ is

$$
\mathbb{T}_{z} \operatorname{Sec}_{k}(X)=\left\langle\mathbb{T}_{p_{0}} X, \ldots, \mathbb{T}_{p_{k}} X\right\rangle .
$$

If $X$ is $k$-defective, then the general hyperplane $H$ containing $\mathbb{T}_{z} \operatorname{Sec}(X)$ is tangent to $X$ along a variety $\Sigma\left(p_{0}, \ldots, p_{k}\right)$ of pure, positive dimension containing $p_{0}, \ldots, p_{k}$.

The final statement suggests the following definition.

Definition $1.10([\mathrm{CC}])$. A variety $X \subset \mathbb{P}^{N}$ is $k$-weakly defective if the general hyperplane, tangent at $(k+1)$ general points of $X$, is tangent along a positive dimensional subvariety through the tangent points. 
Remark 1.11. The Terracini Lemma shows that $k$-defective varieties are also $k$ weakly defective. The converse is not true in general, but weak defect is useful to study defective varieties; see [CC]. Note that if $X$ is not weakly defective, then the hyperplane is tangent exactly at the $(k+1)$ points and has ordinary double points there [CC, Theorem 1.4].

\section{From uniqueness to Singularities}

Let $\nu_{d}\left(\mathbb{P}^{n}\right) \subset \mathbb{P}^{N}$ be the $d$-uple Veronese embedding of $\mathbb{P}^{n}$. We already observed (see Ci] for a deeper account) that the problem of finding the minimal number of linear forms needed to express a general form of degree $d$ is equivalent to determining the dimension of $\operatorname{Sec}_{k}\left(\nu_{d}\left(\mathbb{P}^{n}\right)\right)$. A general form $f$ of degree $d$ is just a general point $p \in \mathbb{P}^{N}$. The number of representations of $f$ as a sum of $(k+1)$ powers of linear forms correspond to the number of $(k+1)$-secant linear spaces to $\nu_{d}\left(\mathbb{P}^{n}\right)$ passing through $p$. If there is just one we will say that there exists the canonical form. Note that $N=\left(\begin{array}{c}d+n \\ n\end{array}\right)-1$ and $\operatorname{dim} \operatorname{Sec}_{k}\left(\nu_{d}\left(\mathbb{P}^{n}\right)\right)=(n+1)(k+1)-1$; therefore there are finitely many representations only if $k+1=\frac{\left(\begin{array}{c}d+n \\ n\end{array}\right)}{n+1}$ is an integer.

Assume that $k+1=\frac{\left(\begin{array}{c}d+n \\ n\end{array}\right)}{n+1}$ is an integer. Consider the natural map

$$
\pi_{1}: \operatorname{Sec}_{k}\left(\nu_{d}\left(\mathbb{P}^{n}\right)\right) \rightarrow \mathbb{P}^{n}
$$

Then the canonical form exists only if $\pi_{1}$ is dominant and birational. The following is a generalization of [CMR, Theorem 4.1]; see also [CR, Theorem 2.6].

Theorem 2.1. Let $X \subset \mathbb{P}^{N}$ be an irreducible variety of dimension $n$. Assume that the natural map $\sigma: \operatorname{Sec}_{k}(X) \rightarrow \mathbb{P}^{N}$ is dominant and birational. Let $z \in \operatorname{Sec}_{k-1}(X)$ be a general point. Consider $\varphi: \mathbb{P}^{N} \rightarrow \mathbb{P}^{n}$ the projection from the embedded tangent space $\mathbb{T}_{z} \operatorname{Sec}_{k-1}(X)$. Then $\varphi_{\mid X}: X \rightarrow \mathbb{P}^{n}$ is dominant and birational.

Proof. Choose a general point $z$ on a general $k$-secant $\mathbb{P}^{k}=\left\langle p_{0}, \ldots, p_{k-1}\right\rangle$. Let $f: Y \rightarrow \mathbb{P}^{N}$ be the blow up of $\operatorname{Sec}_{k-1}(X)$ with exceptional divisor $E$ and fiber $F_{z}=f^{-1}(z)$. Let $y \in F_{z}$ be a general point. This point uniquely determines a linear space $\Pi$ of dimension $k(n+1)$ that contains $\mathbb{T}_{z} \operatorname{Sec}_{k-1}(X)$. Then the projection $\varphi_{\mid X}: X \rightarrow \mathbb{P}^{n}$ is birational if and only if $\left(\Pi \backslash \mathbb{T}_{z} \operatorname{Sec}_{k-1}(X)\right) \cap X$ consists of just one point.

Assume that there exist two points $x_{1}$ and $x_{2}$ in $\left(\Pi \backslash \mathbb{T}_{z} \operatorname{Sec}_{k-1}(X)\right) \cap X$. By the Terracini Lemma, Theorem 1.9.

$$
\mathbb{T}_{z} \operatorname{Sec}_{k-1}(X)=\left\langle\mathbb{T}_{p_{0}} X, \ldots, \mathbb{T}_{p_{k-1}} X\right\rangle .
$$

Consider the linear spaces $\Lambda_{1}=\left\langle x_{1}, p_{0}, \ldots, p_{k-1}\right\rangle$ and $\Lambda_{2}=\left\langle x_{2}, p_{0}, \ldots, p_{k-1}\right\rangle$. The Trisecant Lemma (see for instance [CC, Proposition 2.6]) yields $\Lambda_{1} \neq \Lambda_{2}$. Let $\Lambda_{1}^{Y}$, $\Lambda_{2}^{Y}$ and $\Pi^{Y}$ be the strict transforms on $Y$. Since $z \in\left\langle p_{0}, \ldots, p_{k-1}\right\rangle$ and $y=\Pi^{Y} \cap F_{z}$ then both $\Lambda_{1}^{Y}$ and $\Lambda_{2}^{Y}$ contain the point $y \in F_{z}$. In particular we have

$$
\Lambda_{1}^{Y} \cap \Lambda_{2}^{Y} \neq \emptyset .
$$

Let $\pi_{1}: \operatorname{Sec}_{k}(X) \rightarrow \mathbb{P}^{N}$ be the morphism from the abstract secant variety, and let $\mu: U \rightarrow Y$ be the induced morphism. That is, $U=\operatorname{Sec}_{k}(X) \times_{\mathbb{P}^{N}} Y$. Then there 
exists a commutative diagram

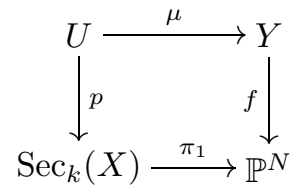

Let $\lambda_{i}$ and $\Lambda_{i}^{U}$ be the strict transform of $\Lambda_{i}$ in $\operatorname{Sec}_{k}(X)$ and $U$, respectively. By Remark $1.8 \lambda_{1} \cap \lambda_{2}=\emptyset$, so that

$$
\Lambda_{1}^{U} \cap \Lambda_{2}^{U}=\emptyset .
$$

This proves that $\mu^{-1}$ is not defined on $y$, a general point of the divisor $E$. That is $\mu$, and henceforth $\pi_{1}$, are not birational.

Remark 2.2. A variety $X^{n} \subset \mathbb{P}^{N}$ is said to have one apparent $(h+1)$-tuple $\mathbb{P}^{h}$ if there exists and is unique a $(h+1)$-secant $\mathbb{P}^{h}$ through the general point of $\mathbb{P}^{N}$; for wider discussions and properties of these varieties we recommend $\mathrm{CR}$. In this dictionary Theorem 2.1 proves that a variety with one apparent $(h+1)$-tuple $\mathbb{P}^{h}$ is rational. This extends the result of [CMR for varieties with one apparent double point; see also $\mathrm{CR}$.

Let $k+1=\frac{\left(\begin{array}{c}d+n \\ n\end{array}\right)}{n+1}$ be an integer and $\varphi: \mathbb{P}^{N} \rightarrow \mathbb{P}^{n}$ the projection from a general tangent space to $\operatorname{Sec}_{k-1}\left(\nu_{d}\left(\mathbb{P}^{n}\right)\right)$. Theorem 2.1 says that the canonical form exists only if $\varphi_{\mid \nu_{d}\left(\mathbb{P}^{n}\right)}$ is birational. Terracini Lemma shows that $\varphi_{\mid \nu_{d}\left(\mathbb{P}^{n}\right)}$ is the map induced by $\mathcal{G}_{d, n, k}$. Therefore to have the canonical form the linear system $\mathcal{G}_{d, n, k}$ has to be expected and effective, and the map associated to $\mathcal{G}_{d, n, k}$ has to be birational onto $\mathbb{P}^{n}$. The final brick of the bridge is the original statement of Noether-Fano inequalities, reinterpreted in modern terminology.

Theorem 2.3 (Noether-Fano inequality $[\mathrm{Co}]$ ). Let $\mathcal{L}$ be a linear system, without fixed components, of forms of degree $d$ on $\mathbb{P}^{n}$. Assume that $\operatorname{dim} \mathcal{L}=n$ and the rational map associated to $\mathcal{L}$ is birational. Then $\left(\mathbb{P}^{n},(n+1) / d \mathcal{L}\right)$ has noncanonical singularities.

We have proved the following necessary condition.

Proposition 2.4. Let $f$ be a general homogeneous form of degree $d$ in $n+1$ variables, with $n \geq 2$. Then $f$ is expressible as the sum of $(k+1)$ powers of linear forms in a unique way only if the pair $\left(\mathbb{P}^{n},(n+1) / d \mathcal{G}_{d, n, k}\right)$ has noncanonical singularities, where $k=\frac{\left(\begin{array}{c}d+n \\ n\end{array}\right)}{n+1}-1$ is an integer.

To test this condition we have to understand the singularities of the linear system $\mathcal{G}_{d, n, k}$. We will do this by a degeneration argument. First we have to establish some good degenerations. This is the content of the following section.

\section{Numerical CONDITIONS TO BE wIn}

In this section we establish sufficient conditions for a linear system of type $\mathcal{H}$ to be wIn. This is just a, maybe pedantic, way to break the main induction argument into small pieces. 
Lemma 3.1. Let $H \subset \mathbb{P}^{n}$ be a hyperplane and assume that $\mathcal{G}_{d, n, l}, \mathcal{G}_{d-1, n, l-h}$, are expected and effective and $\mathcal{G}_{d, n-1, h}$ is expected. Assume further that

$$
\left(\begin{array}{c}
n+d-1 \\
n
\end{array}\right)-(n+1)(l-h)-h \geq \max \left\{1, \operatorname{dim} \mathcal{G}_{d-2, n, l-h}\right\} .
$$

\section{Then $\mathcal{H}_{H, d, n, l, h}$ is wIn.}

Proof. Let $X=P \cup Q$ be as in Definition 1.2, where $Q=\bigcup_{1}^{h} q_{j} \subset H$. By hypothesis $\mathcal{G}_{d, n, l}$ is expected and effective, and by semi-continuity we have to prove that

$$
\operatorname{dim} \mathcal{H}_{H, d, n, l, h}+1 \leq\left(\begin{array}{c}
n+d \\
n
\end{array}\right)-(n+1) l .
$$

Let

$$
h(d-1)=\operatorname{dim} H^{0}\left(\mathbb{P}^{n}, \mathcal{O}_{\mathbb{P}^{n}}(d-1) \otimes \mathcal{I}_{\tilde{X}}\right)
$$

and

$$
h(n-1)=\operatorname{dim} H^{0}\left(\mathbb{P}^{n-1}, \mathcal{O}_{\mathbb{P}^{n-1}}(d) \otimes \mathcal{I}_{Q^{2}}\right) .
$$

By sequence (10) we have

$$
\operatorname{dim} \mathcal{H}_{H, d, n, l, h}+1 \leq h(d-1)+h(n-1) .
$$

By hypothesis $\mathcal{G}_{d, n-1, h}$ is expected therefore

$$
h(n-1)=\left(\begin{array}{c}
n-1+d \\
n-1
\end{array}\right)-n h .
$$

The claim is therefore equivalent to prove that

$$
h(d-1)=\left(\begin{array}{c}
n+d-1 \\
n
\end{array}\right)-(n+1)(l-h)-h>0 .
$$

Indeed this yields

$$
\operatorname{dim} \mathcal{H}_{H, d, n, l, h}+1 \leq\left(\begin{array}{c}
n+d-1 \\
n
\end{array}\right)+\left(\begin{array}{c}
n-1+d \\
n-1
\end{array}\right)-(n+1) l=\left(\begin{array}{c}
n+d \\
n
\end{array}\right)-(n+1) l
$$

and $\operatorname{dim}\left|\mathcal{H}_{H, d, n, l, h} \otimes \mathcal{I}_{H}\right|=h(d-1)-1 \geq 0$. As a consequence we get the exactness of sequence (11).

By hypothesis $\mathcal{G}_{d-1, n, l-h}$ is expected and effective, that is,

$$
\operatorname{dim} \mathcal{G}_{d-1, n, l-h}=\left(\begin{array}{c}
n+d-1 \\
n
\end{array}\right)-(n+1)(l-h) \geq 0 .
$$

Hence to prove (4) it is enough to show that the $h$ points on $H$ impose independent conditions to $\mathcal{G}_{d-1, n, l-h}$. This is equivalent to asking that

$$
H \not \subset \mathrm{Bsl}\left|\mathcal{I}_{P^{2}}(d-1) \otimes\left(\bigotimes_{j=1}^{h-1} \mathcal{I}_{q_{j}}\right)\right|
$$

(the $h-1$ is not a misprint). That is to say,

$$
\left(\begin{array}{c}
n+d-1 \\
n
\end{array}\right)-(n+1)(l-h)-(h-1)>\operatorname{dim} \mathcal{G}_{d-2, n, l-h} .
$$

We can therefore conclude by (2) that

$$
h(d-1)=\left(\begin{array}{c}
n+d-1 \\
n
\end{array}\right)-(n+1)(l-h)-h>0 .
$$


Next we translate the expected and effective conditions of Lemma 3.1 into numerical conditions.

Lemma 3.2. Fix integers $n \geq 3, d \geq 3, l>h$, and a hyperplane $H \subset \mathbb{P}^{n}$, together with the following conditions:

(L) $l<\left\lceil\frac{\left(\begin{array}{c}n+d \\ n\end{array}\right)}{n+1}\right\rceil$,

(H) either $h<\left\lceil\frac{\left(\begin{array}{c}n-1+d \\ n-1\end{array}\right)}{n}\right\rceil$ or $h=\frac{\left(\begin{array}{c}n-1+d \\ n-1\end{array}\right)}{n}$ and $\mathcal{G}_{d, n-1, h}$ is expected,

(LH) $l-h<\left\lceil\frac{\left(\begin{array}{c}n+d-1 \\ n\end{array}\right)}{n+1}\right\rceil$,

(C) $\left(\begin{array}{c}n+d-1 \\ n\end{array}\right)-(n+1)(l-h)-h>0$,

(D4) $l-h>n$,

(D3) $l<\frac{\left(\begin{array}{c}n+3 \\ n\end{array}\right)}{n+1}-\frac{n+2}{3}+1$ and $h=l-1$.

Then the linear system $\mathcal{H}_{H, d, n, l, h}$ is wIn if one of the following set of conditions is satisfied:

- $d \geq 5$ and $(\mathrm{L}),(\mathrm{H}),(\mathrm{LH}),(\mathrm{C})$,

- $d=4$ and $(\mathrm{L}),(\mathrm{H}),(\mathrm{LH}),(\mathrm{C}),(\mathrm{D} 4)$,

- $d=3$ and (D3).

Proof. We will check that the hypothesis of Lemma 3.1 is satisfied for each set of conditions. Hypothesis $(\mathrm{L})$ and $(\mathrm{H})$ together with Theorem 1.4 ensure that $\mathcal{G}_{d, n, l}$ is expected and effective, and $\mathcal{G}_{d, n-1, h}$ is expected.

First note that for $d=3$ condition (D3) forces condition $(\mathrm{L}),(\mathrm{H})$, and $(\mathrm{C})$. Indeed we have

$$
h<\frac{\left(\begin{array}{c}
n+3 \\
n
\end{array}\right)}{n+1}-\frac{n+2}{3}=\frac{(n+3)(n+2)-2(n+2)}{6}=\frac{\left(\begin{array}{c}
n+2 \\
n-1
\end{array}\right)}{n}
$$

and

$$
\left(\begin{array}{c}
n+2 \\
n
\end{array}\right)-(n+1)-\frac{\left(\begin{array}{c}
n+3 \\
n
\end{array}\right)}{n+1}+\frac{n+2}{3} \geq \frac{2(n+2)(n+1)}{6}-(n+1)>0 .
$$

Moreover $\mathcal{G}_{2, n, 1}$ is expected and effective, and $\mathcal{G}_{1, n, 1}$ is empty. Therefore we can apply Lemma 3.1 to conclude.

If $d>3$ condition (LH), together with Theorem 1.4, shows that $\mathcal{G}_{d-1, n, l-h}$ is expected and effective.

If $\mathcal{G}_{d-2, n, l-h}$ is empty, then, by condition (C), we conclude by Lemma 3.1. This is always the case for $d=4$.

Assume that $d \geq 5$ and $\operatorname{dim} \mathcal{G}_{d-2, n, l-h}$ is not empty. If $\mathcal{G}_{d-2, n, h}$ is not expected and effective, then by Theorem $1.4 \operatorname{dim} \mathcal{G}_{d-2, n, l-h}=0$ and by condition (C) we conclude with Lemma 3.1. If $\mathcal{G}_{d-2, n, h}$ is expected and effective, then note that

$$
h \leq\left\lceil\frac{\left(\begin{array}{c}
n-1+d \\
n-1
\end{array}\right)}{n}\right\rceil-1<\left(\begin{array}{c}
n+d-2 \\
n-1
\end{array}\right),
$$

therefore condition $(\mathrm{H})$ yields

$\operatorname{dim} \mathcal{G}_{d-2, n, l-h}=\left(\begin{array}{c}n+d-2 \\ n\end{array}\right)-(n+1)(l-h)-1<\left(\begin{array}{c}n+d-1 \\ n\end{array}\right)-(n+1)(l-h)-h-1$, and we can apply Lemma 3.1 to conclude.

The following lemma allows us to play induction for "small" $l$. Before starting the proof it is convenient to introduce a definition. 
Definition 3.3. For integers $a$ and $b$ let $\lceil a, b\rceil:=\left\lceil\frac{\left(\begin{array}{c}a+b \\ a\end{array}\right)}{a+1}\right\rceil-\frac{\left(\begin{array}{c}a+b \\ a\end{array}\right)}{a+1}$.

Lemma 3.4. Fix a hyperplane $H \subset \mathbb{P}^{n}$. Let

$$
l_{d}:=l=\left\lceil\frac{\left(\begin{array}{c}
n+d+1 \\
n
\end{array}\right)}{n+1}\right\rceil-\left\lceil\frac{\left(\begin{array}{c}
n+d \\
n-1
\end{array}\right)}{n}\right\rceil
$$

and

$$
h_{d}:=h=\left\lceil\frac{\left(\begin{array}{c}
n+d+1 \\
n
\end{array}\right)}{n+1}\right\rceil-\left\lceil\frac{\left(\begin{array}{c}
n+d \\
n-1
\end{array}\right)}{n}\right\rceil-\left\lceil\frac{\left(\begin{array}{c}
n+d \\
n
\end{array}\right)}{n+1}\right\rceil+\left\lceil\frac{\left(\begin{array}{c}
n-1+d \\
n-1
\end{array}\right)}{n}\right\rceil .
$$

Assume that $d \geq 4$ and $n \geq 3$; then $\mathcal{H}_{H, d, n, l, h}$ is wIn.

Proof. Note that $\left(l_{d}-h_{d}\right)=l_{d-1}$. First we simplify the expressions of $h$ and $l$,

$$
\begin{aligned}
h & =\left\lceil\frac{\left(\begin{array}{c}
n+d+1 \\
n
\end{array}\right)}{n+1}\right\rceil-\left\lceil\frac{\left(\begin{array}{c}
n+d \\
n-1
\end{array}\right)}{n}\right\rceil-\left\lceil\frac{\left(\begin{array}{c}
n+d \\
n
\end{array}\right)}{n+1}\right\rceil+\left\lceil\frac{\left(\begin{array}{c}
n-1+d \\
n-1
\end{array}\right)}{n}\right\rceil \\
& =\frac{n\left(\begin{array}{c}
n+d \\
n-1
\end{array}\right)-(n+1)\left(\begin{array}{c}
n+d \\
n-1
\end{array}\right)+(n+1)\left(\begin{array}{c}
n-1+d \\
n-1
\end{array}\right)}{n(n+1)}+\operatorname{Frac}(h) \\
& =\frac{\left(\begin{array}{c}
n-1+d \\
n-1
\end{array}\right)}{n}-\frac{\left(\begin{array}{c}
n+d \\
n-1
\end{array}\right)}{n(n+1)}+\operatorname{Frac}(h),
\end{aligned}
$$

where we collected all the fractional parts in the term

$$
\operatorname{Frac}(h)=\lceil n, d+1\rceil-\lceil n-1, d+1\rceil-\lceil n, d\rceil+\lceil n-1, d\rceil,
$$

in particular $-2<\operatorname{Frac}(h)<2$;

$$
\begin{aligned}
l & =\left\lceil\frac{\left(\begin{array}{c}
n+d+1 \\
n
\end{array}\right)}{n+1}\right\rceil-\left\lceil\frac{\left(\begin{array}{c}
n+d \\
n-1
\end{array}\right)}{n}\right\rceil=\frac{n\left(\begin{array}{c}
n+d+1 \\
n
\end{array}\right)-(n+1)\left(\begin{array}{c}
n+d \\
n-1
\end{array}\right)}{n(n+1)}+\operatorname{Frac}(l) \\
& =\frac{\left(\begin{array}{c}
n+d \\
n
\end{array}\right)}{n+1}-\frac{\left(\begin{array}{c}
n+d \\
n-1
\end{array}\right)}{n(n+1)}+\operatorname{Frac}(l),
\end{aligned}
$$

where the fractional part is

$$
\operatorname{Frac}(l)=\lceil n, d+1\rceil-\lceil n-1, d+1\rceil,
$$

this time $-1<\operatorname{Frac}(l)<1$. Incidentally this shows that both $l$ and $l-h$ are positive integers.

The plan is to prove the claim by checking the conditions of Lemma 3.2

Condition $(\mathrm{H})$ in Lemma 3.2 is given by (5) and the inequality

$$
\left(\begin{array}{l}
n+d \\
n-1
\end{array}\right) \geq 2 n(n+1)
$$

which is true for any $d \geq 4$ and $n \geq 4$. In the missing case, namely $(n, d)=(3,4)$, we have $h=3<5=\left\lceil\frac{\left(\begin{array}{c}6 \\ 2\end{array}\right)}{3}\right\rceil$.

Condition (L) is obtained by (77) and the inequality

$$
\left(\begin{array}{l}
n+d \\
n-1
\end{array}\right) \geq n(n+1)
$$

which is verified for any $d \geq 3$ and $n \geq 3$.

As observed condition (LH) is just condition (L) for $d-1$. 
To check condition (C) note that by (5) and (7)

$$
\begin{aligned}
\left(\begin{array}{c}
n+d-1 \\
n
\end{array}\right)-(n+1)(l-h)-h & =\left(\begin{array}{c}
n+d-1 \\
n
\end{array}\right)-(n+1) l+n h \\
& =\frac{\left(\begin{array}{c}
n+d \\
n-1
\end{array}\right)}{n}-\frac{\left(\begin{array}{c}
n+d \\
n-1
\end{array}\right)}{n+1}-(n+1) \operatorname{Frac}(l)+n \operatorname{Frac}(h) \\
& \geq \frac{\left(\begin{array}{l}
n+d \\
n-1
\end{array}\right)}{n(n+1)}-(n\lceil n, d\rceil+\lceil n, d+1\rceil) .
\end{aligned}
$$

The requested bound is therefore implied by

$$
\left(\begin{array}{l}
n+d \\
n-1
\end{array}\right) \geq n(n+1)^{2}
$$

This is verified for $d \geq 4$ and $n \geq 7$ or $d \geq 5$ and $n \geq 5$ or $d \geq 6$ and $n \geq 4$ or $d \geq 8$ and $n \geq 3$. The remaining cases can be checked by a direct computation. Let $\delta=\left(\begin{array}{c}n+d-1 \\ n\end{array}\right)-(n+1)(l-h)-h$; then

\begin{tabular}{|c|cccccccc|}
\hline$(d, n)$ & $(4,6)$ & $(4,5)$ & $(4,4)$ & $(4,3)$ & $(5,4)$ & $(5,3)$ & $(6,3)$ & $(7,3)$ \\
\hline$l-h$ & 9 & 7 & 5 & 4 & 12 & 7 & 11 & 18 \\
\hline$h$ & 15 & 9 & 7 & 3 & 9 & 4 & 7 & 9 \\
\hline \hline$\delta$ & 6 & 5 & 3 & 1 & 1 & 3 & 5 & 3 \\
\hline
\end{tabular}

If $d=4$ we have to prove (D4), by definition

$$
l-h=\left\lceil\frac{\left(\begin{array}{c}
n+4 \\
n
\end{array}\right)}{n+1}\right\rceil-\left\lceil\frac{\left(\begin{array}{c}
n+3 \\
n-1
\end{array}\right)}{n}\right\rceil>\frac{\left(\begin{array}{c}
n+3 \\
n
\end{array}\right)}{n+1}-\frac{\left(\begin{array}{c}
n+3 \\
n-1
\end{array}\right)}{n(n+1)}-1 .
$$

Expanding the binomial we get

$$
l-h>\frac{(n+3)(n+2)}{8}-1
$$

The requested bound is therefore implied by

$$
(n+3)(n+2) \geq 8(n+1) .
$$

This is verified for $n \geq 4$. For $n=3$ we have $l-h=9-5>3=n$.

\section{Singularities of Linear Systems}

This is the unique result we have about singularities of cubic forms.

Theorem 4.1. Let $G \in \mathcal{G}_{d, n, l}$ be a general element. Assume that either

- $d \geq 4, n \geq 3$, and $l=\left\lceil\frac{\left(\begin{array}{c}n+d+1 \\ n\end{array}\right)}{n+1}\right\rceil-\left\lceil\frac{\left(\begin{array}{c}n+d \\ n-1\end{array}\right)}{n}\right\rceil$, or

- $d=3, n \geq 3$ and $l<\frac{\left(\begin{array}{c}n+3 \\ n\end{array}\right)}{n+1}-\frac{n+2}{3}+1$.

Then $\mathcal{G}_{d, n, l}$ is expected and effective, and $G$ has only ordinary double points.

Remark 4.2. As in the interpolation problem cubics are rather difficult because the degeneration technique does not work for the highest possible values of $l$. Nevertheless this is what is needed to conclude for degree at least 4.

Proof. We prove the claim by induction on $d$.

Assume that $d \geq 4$. Let $l^{\prime}=\left\lceil\frac{\left(\begin{array}{c}n+d \\ n\end{array}\right)}{n+1}\right\rceil-\left\lceil\frac{\left(\begin{array}{c}n+d-1 \\ n-1\end{array}\right)}{n}\right\rceil$ and $h=l-l^{\prime}$. Fix a general hyperplane $H \subset \mathbb{P}^{n}$; then by Lemma $3.4 \mathcal{H}_{H, d, n, l, h}$ is wIn. This proves that $\mathcal{G}_{d, n, l}$ is expected and effective. Since $\left|\mathcal{H}_{H, d, n, l, h} \otimes \mathcal{I}_{H}\right| \neq \emptyset$ we can look at the linear 
system $\mathcal{H}_{H, d, n, l, h}$ from a different viewpoint. Choose $l-h$ general points in $\mathbb{P}^{n}$, $p_{1}, \ldots, p_{l-h}$. Let

$$
D \in \mathcal{G}_{d-1, n, l-h}
$$

be a general element. Choose a general hyperplane $H \subset \mathbb{P}^{n}$ and $h$ general points on $C=D \cap H, q_{1}, \ldots, q_{h}$.

By induction hypothesis $D$ has only ordinary double points. Therefore the general element of $\mathcal{H}_{H, d, n, l, h}$ has only isolated singularities at the $p_{i} \mathrm{~s}$, and by Corollary 1.6 the general element of $\mathcal{G}_{d, n, l}$ has only ordinary double points.

To conclude we need to prove the first step of the induction. If $d=3$ one gets

$$
l=\left\lceil\frac{\left(\begin{array}{c}
n+4 \\
n
\end{array}\right)}{n+1}\right\rceil-\left\lceil\frac{\left(\begin{array}{c}
n+3 \\
n-1
\end{array}\right)}{n}\right\rceil=\frac{(n+3)(n+2)}{8}+\lceil n+4, n\rceil-\lceil n+3, n-1\rceil
$$

and

$$
l-\frac{\left(\begin{array}{c}
n+3 \\
n
\end{array}\right)}{n+1}+\frac{n+2}{3}-1<\frac{(n+2)(5-n)}{24} .
$$

This proves condition (D3) for $n \geq 5$. Therefore $\mathcal{H}_{H, 3, l, l-1}$ is wIn, and there is at least one divisor $D \in \mathcal{H}_{H, 3, l, l-1}$ with $D=Q+H$ with $Q$ a quadric cone. This, together with Corollary 1.6. proves the theorem for $n \geq 5$. For $n=3,4$ note that $l$ is, respectively, at most 4 and 5 . Then we do not need any specialization. The linear system $\mathcal{G}_{d, n, l}$ always contains a reducible divisor $Q+H$ where $Q$ is a quadric cone.

We are now ready to prove the main result about forms with assigned singularities.

Theorem 4.3. Assume that $n \geq 3$ and $d \geq 3$ and either

$$
\begin{aligned}
& \text { - } l_{0}=\left\lceil\frac{\left(\begin{array}{c}
n+d+1 \\
n
\end{array}\right)}{n+1}\right\rceil-1 \text { and } n\lceil n-1, d+1\rceil-(n+1)\lceil n, d+1\rceil+1>0 \text {, or } \\
& \text { - } l_{1}=\left\lceil\frac{\left(\begin{array}{c}
n+d \\
n
\end{array}\right)}{n+1}\right\rceil-2 \text { or } \\
& \text { - } l_{2}=\left\lceil\frac{\left(\begin{array}{c}
n+d+1 \\
n
\end{array}\right.}{n+1}\right\rceil-1,\lceil n-1, d+1\rceil=0 \text { and either } d \geq 4 \text { or } d=3 \text { and } n \geq 6 \text {. }
\end{aligned}
$$

Then the general element in $\mathcal{G}_{d+1, n, l_{i}}$ has only ordinary double points.

Remark 4.4. The $n=2$ case is well known, $[\mathrm{AC}$. We will recall it in Lemma 5.1. Note that for $n=2, d=6$, and $l=9$ the unique 6 -ic with 9 general double points is a double cubic. For $n=3$ there is another exception. Consider $\mathcal{G}_{4,3,8}$. A dimension count shows that $\mathcal{G}_{4,3,8}=\left\{Q_{1}^{2}, Q_{2}^{2}, Q_{1} Q_{2}\right\}$, where $Q_{i}$ are the quadrics passing simply through the 8 points. In particular the general element of $\mathcal{G}_{4,3,8}$ has a curve of singularities. We believe that there are very few expected and effective linear systems $\mathcal{G}_{d, n, l}$ with nonisolated singularities. Maybe these are the only ones 1 It would be interesting to classify them all; see Corollary 4.7 for a partial result in this direction. Note that all linear systems that are not expected have nonisolated singularities.

Corollary 4.5. The Veronese embedding $\nu_{d}\left(\mathbb{P}^{n}\right) \subset \mathbb{P}^{N}$ is not $k$-weakly defective for $d \geq 4$ and $n \geq 3$ if $\operatorname{cod} \operatorname{Sec}_{k}\left(\nu_{d}\left(\mathbb{P}^{n}\right)\right) \geq n+1$.

Remark 4.6. Luca Chiantini and Ciro Ciliberto pointed out the following consequence of nonweakly defectiveness of the Veronese embedding. Assume that $\nu_{d}\left(\mathbb{P}^{n}\right)$

\footnotetext{
${ }^{1}$ Edoardo Ballico informed the author that he gave a positive answer for $d \geq 4$ in $\mathrm{Ba}$.
} 
is not $k$-weakly defective. Let $x \in \nu_{d}\left(\mathbb{P}^{n}\right)$ be a general point. Then, by the Terracini Lemma, there exists a unique $\mathbb{P}^{k}$ which is $(k+1)$-secant through $x$. That is, the natural map $\pi_{k}: \operatorname{Sec}_{k}\left(\nu_{d}\left(\mathbb{P}^{n}\right)\right) \rightarrow \mathbb{P}^{N}$ is birational onto the image. This improves results of [IK, Theorems 2.6, 7.18], where this statement was proved for $k \sim\left(\begin{array}{c}n+d / 2 \\ n\end{array}\right)$.

Proof of Theorem 4.3. Let $h_{0}=h_{1}=\left\lceil\frac{\left(\begin{array}{c}n+d \\ n-1\end{array}\right)}{n}\right\rceil-1$ and $h_{2}=\frac{\left(\begin{array}{c}n+d \\ n-1\end{array}\right)}{n}$ be integers. Note that if either $d \geq 4$ or $d=3$ and $n \geq 6$, then by Theorem 1.4 the linear system $\mathcal{G}_{d+1, n-1, h_{2}}$ is expected. Theorem 4.1] shows that the general element $D \in \mathcal{G}_{d, n, l_{i}-h_{i}}$ has only ordinary double points. Consider the linear systems $\mathcal{H}_{H, d+1, n, l_{i}, h_{i}}$. By hypothesis we have

$$
\left(\begin{array}{c}
n+d \\
n
\end{array}\right)-(n+1)\left(l_{0}-h_{0}\right)-h_{0}=n\lceil n-1, d+1\rceil-(n+1)\lceil n, d+1\rceil+1>0
$$

and, for $i=1,2$

$$
\left(\begin{array}{c}
n+d \\
n
\end{array}\right)-(n+1)\left(l_{i}-h_{i}\right)-h_{i}=(n+1)+n\lceil n-1, d+1\rceil-(n+1)\lceil n, d+1\rceil+1>0 .
$$

This proves, via Lemma 3.2, that $\mathcal{H}_{H, d+1, n, l_{i}, h_{i}}$ is wIn, for $d \geq 4$. For $d=3$ note that

$$
\begin{aligned}
l_{i}-h_{i} & >\frac{n\left(\begin{array}{c}
n+4 \\
n
\end{array}\right)-(n+1)\left(\begin{array}{c}
n+3 \\
n-1
\end{array}\right)}{n(n+1)}-2=\frac{n\left(\begin{array}{c}
n+3 \\
n
\end{array}\right)-\left(\begin{array}{c}
n+3 \\
n-1
\end{array}\right)}{n(n+1)}-2 \\
& =\frac{(n+3)(n+2)}{8}-2
\end{aligned}
$$

for $i=0,1,2$. Then $l_{i}-h_{i}>n$ for $n \geq 5$ and, via Lemma 3.2, $\mathcal{H}_{H, d+1, n, l_{i}, h_{i}}$ is wIn in these cases. The leftover has to be checked directly. If $(d, n)=(3,4)$ we have $l_{0}-1=l_{1}=12$ and $h_{1}=8$. If $(d, n)=(3,3)$ we have $l_{1}=7, h_{1}=3$. Therefore we can apply Lemma 3.2 for $\mathcal{H}_{H, 4,3, l_{i}, h_{i}}$, with $i=0,1$ and $\mathcal{H}_{H, 4,4, l_{0}, h_{0}}$. For $\mathcal{H}_{H, 4,4, l_{1}, h_{1}}$ note that

$$
\left(\begin{array}{c}
n+3 \\
n
\end{array}\right)-(n+1)\left(l_{1}-h_{1}\right)-h_{1}=7>\operatorname{dim} \mathcal{G}_{2,4,4}=1 .
$$

This time we use Lemma 3.1 to conclude that $\mathcal{H}_{H, 4,4, l_{1}, h_{1}}$ is wIn.

We can, therefore, assume that $D+H \in \mathcal{H}_{H, d+1, n, l_{i}, h_{i}}$ for $D \in \mathcal{G}_{d, n, l_{i}-h_{i}}$ with only ordinary double points. This together with Corollary 1.6 conclude the proof.

We can use the above theorem to determine the singularities of $\mathcal{G}_{d, n, l}$ for $d \geq 5$ prime and $d=4$; keep in mind Remark4.4.

Corollary 4.7. Assume that $\mathcal{G}_{d, n, l}$ is effective. Then the general element $G \in \mathcal{G}_{d, n, l}$ has ordinary double points if either $d=4$, and $(n, l) \neq(3,8),(3,9)$ or $d \geq 5$ is a prime. We already described the exceptions.

Proof. Note that

$$
\frac{\left(\begin{array}{c}
n+d \\
n
\end{array}\right)}{n+1}=\frac{(n+d)(n+d-1) \cdot \ldots \cdot(n+2)}{d(d-1) !}
$$

Note that both

$$
\frac{(n+d)(n+d-1) \cdot \ldots \cdot(n+2)}{(d-1) !}
$$


and

$$
\frac{(n-1+d)(n-1+d-1) \cdot \ldots \cdot(n+1)}{(d-1) !}
$$

are integers. Furthermore for some $1 \leq a \leq d$ we have $n+a \equiv 0(d)$. Assume that $d$ is a prime. Then either $\lceil n, d\rceil$ or $\lceil n-1, d\rceil$ vanishes, and we can apply Theorem 4.3 for any triple $(d, n, l)$ with $\mathcal{G}_{d, n, l}$ effective.

Assume that $d=4$, this time

$$
\frac{\left(\begin{array}{c}
n+4 \\
n
\end{array}\right)}{n+1}=\frac{(n+4)(n+3)(n+2)}{4 \cdot 3 \cdot 2} .
$$

If $n+2 \equiv 0(2)$ we have $(n+4)(n+3)(n+2) \equiv 0(24)$, while if $n+2 \equiv 1(2)$ we have $(n+3)(n+2)(n+1) \equiv 0(24)$. This yields that either $\lceil n, 4\rceil=0$ or $\lceil n-1,4\rceil=0$. This proves the claim, by Theorem 4.3. for $n \geq 6$. If $n=2,4,5$ it is easy to check that $\lceil n, 4\rceil=0$. This concludes the proof.

Remark 4.8. Note that for $(d, n, l)=(6,9,500)$ the conditions of Theorem 4.3 are not satisfied. This is the first occurrence that we cannot treat in degree 6 . We have no statement, similar to Corollary 4.7, for fixed $n$ and any $d$.

\section{Proof of Theorem 1}

We are now in a condition to prove Theorem 1. The following lemma allows us to treat the two-dimensional case.

Lemma $5.1([\mathrm{AC}])$. Let $l=\left\lceil\frac{\left(\begin{array}{c}d+2 \\ 2\end{array}\right)}{3}\right\rceil-1$. Then $\left(\mathbb{P}^{2}, 1 / 2 \mathcal{G}_{d, n, l}\right)$ has canonical singularities.

Proof. Let $G \in \mathcal{G}_{d, n, l}$ be a general element. Then by [CC, Theorem 1.4] either $G$ has only the imposed ordinary double points, or it has a fixed component of singularities passing through $P$. The latter is easily seen, by a dimension count, to be possible only for $d=6$ and $l=9$. In this special case the unique 6 -ic with assigned 9 double points is a double cubic. Thus $G$ has only double points and $\left(\mathbb{P}^{2}, 1 / 2 \mathcal{G}_{d, n, l}\right)$ has canonical singularities.

Proof of Theorem 1. We already observed (Proposition 2.4) that for the existence of the canonical form,

$$
k+1=\frac{\left(\begin{array}{c}
d+n \\
n
\end{array}\right)}{n+1}
$$

has to be an integer, and $\left(\mathbb{P}^{n},(n+1) / d \mathcal{G}_{d, n, k}\right)$ cannot be canonical.

If $n=2$, this means that either $d=4,5$ or $d \geq 7$. If $d=4$, we have $k=4$, and $d^{2}=16=4 k$. In particular the map associated to $\mathcal{G}_{4,2,4}$ is composed with a pencil. Note that $\operatorname{dim} \mathcal{G}_{4,2,5}=0$ and $\mathcal{G}_{4,2,5}$ is not expected. If $d=5$, we have $k=6$ and $d^{2}=25=4 k+1$. It is not difficult to see that the scheme base locus of $\mathcal{G}_{5,2,6}$ is just $P^{2}$. In this case we already know that there exists a canonical form, [Hi]. Assume that $d \geq 7$; then [AC, Theorem 3.2] shows that $\mathcal{G}_{d, 2, k}$ does not have fixed components, and by Lemma $5.1\left(\mathbb{P}^{2}, 3 / d \mathcal{G}_{d, 2, k}\right)$ has canonical singularities. Therefore uniqueness is impossible.

If $n \geq 3$, then by Theorem 4.3 the general element $G \in \mathcal{G}_{d, n, k}$ has only ordinary double points. In particular the divisor $G$ is irreducible, and after the blow up of the singular points it is smooth. This proves that the log pair $\left(\mathbb{P}^{n},(n+1) / d \mathcal{G}_{d, n, k}\right)$ has canonical singularities and concludes the proof. 
Remark 5.2. To extend Theorem 1 to a lower degree one has to study the base locus of $\mathcal{G}_{d, n, l}$ and not only its singularities. The semi-continuity works for the dimension of the Base Locus. Furthermore, with a similar argument it is possible to prove that functions $\psi_{i}$ of Lemma 1.5 are zero at least for "small" $l$. What is completely missing is a way to determine the scheme base locus out of this information.

Theorem 1 together with the known exceptions allow us to answer the uniqueness question for forms of at most 4 variables.

Corollary 5.3. Let $f$ be a homogeneous form of degree $d$ in $n+1$ variables. If $n \leq 3$, then canonical form exists if and only if $(d, n)=(2 k+1,1),(5,2),(3,3)$.

\section{REFERENCES}

[AH1] J. Alexander, A. Hirschowitz, The blown-up Horace method: application to fourth-order interpolation Invent. Math. 107 (1992), no. 3, 585-602. MR.1150603 (93d:13017)

[AH2] - Polynomial interpolation in several variables J. Algebraic Geom. 4 (1995), no. 2, 201-222. MR1311347 (96f:14065)

[AC] E. Arbarello, M. Cornalba, Footnotes to a paper of Beniamino Segre Math. Ann. 256 (1981), no. 3, 341-362. MR0626954 (83d:14016)

[Ba] E. Ballico, On the weak non-defectivity of Veronese embeddings of projective spaces, Cent. Eur. J. Math. 3 (2005), no. 2, 183-187. MR2129920

[Ch1] K. Chandler, A brief proof of a maximal rank theorem for generic double points in projective space Trans. Amer. Math. Soc. 353 (2001), no. 5, 1907-1920. MR1813598 (2002i:14046)

[Ch2] Linear systems of cubics singular at general points of projective space Comp. Math. 134 (2002), no. 3, 269-282. MR1943904 (2003i:13023)

[CC] L. Chiantini, C. Ciliberto Weakly defective varieties Trans. Amer. Math. Soc. 354 (2002), no. 1, 151-178. MR1859030 (2003b:14063)

[Ci] C. Ciliberto, Geometric aspects of polynomial interpolation in more variables and of Waring's problem European Congress of Mathematics, Vol. I (Barcelona, 2000), 289-316, Progr. Math., 201, Birkhäuser, Basel, 2001. MR1905326 (2003i:14058)

[CMR] C. Ciliberto, M. Mella, F. Russo, Varieties with one apparent double point J. Algebraic Geom. 13 (2004), 475-512. MR.2047678 (2005b:14078)

[CR] C. Ciliberto, F. Russo Varieties with minimal secant degree and linear systems of maximal dimension on surfaces math.AG/0406494 to appear in Adv. in Math.

[Co] A. Corti, Factoring birational maps of threefolds after Sarkisov J. Algebraic Geom. 4 (1995), no. 2, 223-254. MR1311348 (96c:14013)

[Di] L. Dickson, History of the theory of numbers. Vol. II: Diophantine analysis Chelsea Publishing Co., New York, 1966, xxv+803 pp. MR0245500 (39:6807b)

[Hi] D. Hilbert, Letter adresseé à M. Hermite, Gesam. Abh. vol. II 148-153.

[Ia] A. Iarrobino, Inverse system of a symbolic power. II. The Waring problem for forms J. Algebra 174 (1995), no. 3, 1091-1110. MR.1337187 (96i:13018)

[IK] A. Iarrobino, V. Kanev, Power sums, Gorenstein algebras, and determinantal loci Appendix C by Iarrobino and Steven L. Kleiman. Lecture Notes in Mathematics, 1721, Springer-Verlag, Berlin, 1999. xxxii+345 pp. MR1735271 (2001d:14056)

[IR1] A. Iliev, K. Ranestad, K3 surfaces of genus 8 and varieties of sums of powers of cubic fourfolds Trans. Amer. Math. Soc. 353 (2001), no. 4, 1455-1468. MR1806733 (2002c:14082)

[IR2] Canonical curves and varieties of sums of powers of cubic polynomials J. Algebra 246 (2001), no. 1, 385-393. MR1872627 (2003c:14032)

$[\mathrm{Mu}]$ S. Mukai, Fano 3-folds Complex projective geometry (Trieste, 1989/Bergen, 1989), 255263, London Math. Soc. Lecture Note Ser., 179, Cambridge Univ. Press, Cambridge, 1992. MR1201387 (94a:14042)

[Pa] F. Palatini, Sulla rappresentazione delle forme ternarie mediante la somma di potenze di forme lineari Rom. Acc. L. Rend. 12 (1903) 378-384.

[Ri] H.W. Richmond, On canonical forms Quart. J. Pure Appl. Math. 33 (1904) 967-984. 
[RS] K. Ranestad, F. Schreyer, Varieties of sums of powers J. Reine Angew. Math. 525 (2000), 147-181. MR1780430 (2001m:14009)

[Sy] J.J. Sylvester, Collected works Cambridge University Press, 1904.

[Te] A. Terracini, Sulle $V_{k}$ per cui la varietà degli $S_{h}(h+1)$-seganti ha dimensione minore dell'ordinario, Rend. Circ. Mat. Palermo 31 (1911), 392-396.

Dipartimento di Matematica, Universitì di Ferrara, Via Machiavelli 35, 44100 FerRARA, ITALY

E-mail address: mll@unife.it 